\title{
Relaxation of the one child policy and trends in caesarean section rates and birth outcomes in China between 2012 and 2016: an observational study of nearly seven million health facility births
}

\author{
Liang J, Mu Y, Li X, et al
}

Study question How has the relaxation of the one child policy and policies to reduce caesarean section rates affected trends over time in caesarean section rates in China?

Methods The authors used data on 6838582 births in 438 hospitals recorded through China's National Maternal Near Miss Surveillance System (NMNMSS) between 2012 and 2016. They examined trends over time in caesarean sections using Poisson regression adjusting for changes in the obstetric profile of births over time, and separating trends in nulliparous women, multiparous women without a uterine scare and multiparous women with a uterine scar.

Study answer and limitations The relaxation of the one child policy was associated with an increase in the proportion of multiparous births (from $34.1 \%$ in 2012 to $46.7 \%$ in 2016) and births in women with a uterine scar (from $9.8 \%$ to $17.7 \%$ of all births). Caesarean sections declined noticeably in nulliparous women (adjusted relative risk comparing 2016 with 2012: 0.75 , 95\% confidence interval 0.73 to 0.77 ), but they also declined in multiparous women without a uterine scar $(0.65,0.62$ to 0.77). The decrease in caesarean section rates was most pronounced in hospitals with the highest rates in 2012, consistent with the Chinese government's policy of targeting hospitals with the highest rates.

Since this is not an intervention study it is not possible to attribute the decline in caesarean sections to one particular policy. However, by using a statistical model that adjusted for the main demographic and clinical changes potentially associated with 
Item: BMJ-UK; Article ID: liaj039516;

Article Type: Research paper; TOC Heading: Research; DOI: 10.1136/bmj.k817

the relaxation of the one child policy, we were able to separate the effects of the relaxation of the one child policy from policies specifically aimed at reversing the high caesarean section rate. 
Item: BMJ-UK; Article ID: liaj039516;

Article Type: Research paper; TOC Heading: Research; DOI: 10.1136/bmj.k817

Table Time trends in caesarean section rates in all women and by parity (438 hospitals in China in 2012-16)

\begin{tabular}{lccccc}
\hline Variables & $\mathbf{2 0 1 2}$ & $\mathbf{2 0 1 3}$ & $\mathbf{2 0 1 4}$ & $\mathbf{2 0 1 5}$ & $\mathbf{2 0 1 6}$ \\
\hline Weighted caesarean section & & & & & \\
rate (\%) (No of caesarean & & & & & \\
sections, \% of caesarean & & & & & \\
sections): & & & & & \\
$\quad$ All & $45.3(617460,100.0)$ & $45.6(606607,100.0)$ & $43.8(660154,100.0)$ & $42.1(561127,100.0)$ & $41.1(632753,100.0)$ \\
$\quad$ Nulliparous & $46.6(415530,67.4)$ & $46.7(393807,64.9)$ & $43.3(397980,60.3)$ & $40.5(300413,53.6)$ & $37.9(308308,48.7)$ \\
$\quad$ Multiparous without uterine & $25.4(84711,13.8)$ & $24.9(84600,14.0)$ & $22.7(90494,13.7)$ & $20.5(78339,14.0)$ & $18.5(84100,13.3)$ \\
scar & & & & & \\
$\quad$ Multiparous with uterine & $90.3(116049,18.8)$ & $90.9(127559,21.1)$ & $91.0(171257,26.0)$ & $90.5(181811,32.4)$ & $90.4(239892,37.9)$ \\
scar & & & & & \\
\hline
\end{tabular}


What this study adds China's success in reverting the rising trends in caesarean sections is remarkable given that the demographic change resulting from the relaxation of the one child policy would have led to an increase in the need for caesarean sections. China's experience suggests that change is possible when strategies are comprehensive and deal with the system level factors that underpin overuse as well as the various incentives at work during a clinical encounter.

Funding, competing interests, data sharing The funders (see full paper on bmj.com) had no role in the study design; collection, analysis, and interpretation of data; writing of the report; or decision to submit the article for publication. The authors declare no competing interests. No additional data are available.

Cite this as: $B M J$ 2018;360:k817 